\title{
Comprensión de aspectos epistémicos de la naturaleza de la ciencia y valoración de su dimensión social
}

\author{
Radu Bogdan Toma \\ Departamento de Didácticas Especificas, Universidad de Burgos. Burgos. España.rbtoma@ubu.es \\ ORCID: https:/ / orcid.org/0000-0003-4846-7323
}

[Recibido: 1 Octubre 2019. Revisado: 2 Diciembre 2019. Aceptado: 3 Marzo 2020]

\begin{abstract}
Resumen: La importancia de la naturaleza de la ciencia $(\mathrm{NdC})$ reside en varias suposiciones teóricas que no han sido contrastadas de forma empírica. Este estudio examina la presunción de que una comprensión apropiada algunos aspectos epistémicos de la $\mathrm{NdC}$ estaría relacionada con una mayor valoración de la ciencia, en $6^{\circ}$ curso de Educación Primaria $(N=341)$. Los resultados revelan actitudes más positivas en los estudiantes con concepciones apropiadas de los aspectos «naturaleza provisional» $\mathrm{y}$ «observaciones e inferencias», lo que ofrece apoyo empírico al argumento cultural para la enseñanza de la $\mathrm{NdC}$ en el nivel elemental. Si bien estos hallazgos subrayan el valor educativo de la $\mathrm{NdC}$, se requiere el desarrollo de futuros estudios que adopten una conceptualización de la $\mathrm{NdC}$ que supere las limitaciones de la denominada en la bibliografía como visión de consenso.
\end{abstract}

Palabras clave: actitudes hacia la ciencia, dimensión social de la ciencia, educación científica, Educación Primaria, naturaleza de la ciencia.

Understanding of epistemic aspects of nature of science and appreciation of its social dimension

\begin{abstract}
The importance of nature of science (NOS) rests on several untested assumptions. This study examines the assumption that an informed understanding of some epistemic NOS aspects would be related to a greater appreciation of science, in $6^{\text {th }}$ grade of elementary education $(N=341)$. The results revealed more positive attitudes in students with informed views of the «tentative» and «observation and inferences» aspects of NOS, which offers empirical support for the cultural argument for teaching NOS in elementary education. While these findings underlie the educational value of NOS, there is a need for future studies addressing this aspect through NOS frameworks that overcomes the limitations of the one named in the literature as the consensus view.
\end{abstract}

Keywords: attitudes towards science, social dimension of science, science education, elementary education, nature of science.

Para citar este artículo: Bogdan Toma R. (2020) Comprensión de aspectos epistémicos de la naturaleza de la ciencia y valoración de su dimensión social. Revista Eureka sobre Enseñanza y Divulgación de las Ciencias 17(2), 2303. doi: 10.25267/Rev_Eureka_ensen_divulg_cienc.2020.v17.i2.2303

\section{Introducción}

Desde hace años, la didáctica de las ciencias incluye entre sus objetivos básicos el desarrollo de concepciones adecuadas sobre la naturaleza de la ciencia $(\mathrm{NdC})$. Bajo el argumento de que la ciudadanía debe alcanzar ideas informadas sobre la $\mathrm{NdC}$, este contenido ha sido promovido en informes y reformas educativas internacionales como un factor clave para alcanzar la alfabetización científica de los estudiantes (Olson 2018). Driver, Leach, Millar y Scott (1996) ofrecieron varios argumentos a favor de la $\mathrm{NdC}$ para la alfabetización científica. Específicamente, sostuvieron que una adecuada comprensión de la $\mathrm{NdC}$ es necesaria para (i) gestionar y usar adecuadamente los objetos y procesos tecnológicos presentes en la vida cotidiana (argumento utilitario, p. 16), (ii) dar sentido a las cuestiones socio-científicas y tomar decisiones razonadas en un mundo cada vez más influenciado por los procesos y productos de la ciencia (argumento democrático, p. 18), (iii) apreciar la ciencia como un elemento importante de la cultura contemporánea (argumento cultural, p. 19), (iv) tomar conciencia de las normas de la comunidad científica que encarnan compromisos morales de un valor general para la ciudadanía (argumento moral, p. 19) y, por último, (v) apoyar el aprendizaje de los contenidos científicos (argumento del aprendizaje de la ciencia, p. 20).

\author{
Revista Eureka sobre Enseñanza y Divulgación de las Ciencias \\ Universidad de Cádir. APAC-Eureka. ISSN: 1697-011X \\ bttp:/ / dx.doi.org/10.25267/Rev_Eureka_ensen_divulg_cienc.2020.v17.i2.2303 \\ http:/ / reuredc.uca.es
}




\section{Planteamiento del problema y objetivo de la investigación}

Los didactas de las ciencias experimentales han formulado una amplia gama de motivos para enseñar sobre la $\mathrm{NdC}$. Sin embargo, aunque muchas de estas razones son intuitivas y lógicas desde un punto de vista teórico, existe poca evidencia empírica de que el aprendizaje de la naturaleza de la ciencia produzca los efectos deseados (Lederman 1999). Aunque convincentes y conceptualmente válidos, los argumentos a favor de la enseñanza de la $\mathrm{NdC}$ de Driver et al. (1996) requieren de un análisis empírico más detallado; especialmente a la luz de los estudios que sugieren que la comprensión de la $\mathrm{NdC}$ no tiene un papel primordial en la toma de decisiones (argumento democrático, e.g., Bell y Lederman 2002), ni en la mejora de la comprensión de las teorías científicas (argumento del aprendizaje de la ciencia, e.g., Cofré, Cuevas y Becerra 2017). Asimismo, algunos estudios sobre el argumento cultural han reportado resultados contradictorios; mientras que Harty, Samuel y Andersen (1991) no encontraron ninguna correlación entre la comprensión de la $\mathrm{NdC}$ y las actitudes hacia la ciencia en maestros en formación inicial, Evans y Durant (1995) concluyeron que unos niveles más altos de conocimiento sobre los procesos científicos están asociados con actitudes de mayor apoyo hacia la ciencia, en población adulta. No obstante, al leal saber del autor de este estudio, no existen investigaciones desarrolladas en el contexto español con estudiantes de Educación Primaria (EP) que examinen la relación entre concepciones de la $\mathrm{NdC}$ y actitudes de aprecio de la ciencia como elemento clave de la cultura.

En palabras de McComas (2017), que se refiere al argumento cultual de Driver y colegas, "La $\mathrm{NdC}$ es necesaria para apreciar el valor de la ciencia como parte de la cultura contemporánea" (p. 72). Por consiguiente, dado que la comprensión de la $\mathrm{NdC}$ se postula como vital para fomentar actitudes de apreciación hacia el valor que supone la ciencia en la actual sociedad (Lederman, Bartos y Lederman 2014), en este estudio se explora la presunción de que una comprensión adecuada de la $\mathrm{NdC}$ estaría relacionada con actitudes más positivas hacia la aportación social de la ciencia y su valor en la sociedad.

\section{Fundamentación teórica}

\section{Naturaleza de la Ciencia}

La NdC se refiere a los supuestos, valores y creencias que son inherentes al desarrollo y la validación del conocimiento científico, así como a las características de este cuerpo de conocimiento (Lederman 2007). En las dos últimas décadas, la conceptualización de la $\mathrm{NdC}$ que ha prevalecido sobre qué aspectos de esta enseñar, es la denominada consensus view (visión de consenso), promovida principalmente por Norman G. Lederman y su grupo de investigación (Lederman, Abd-El-Khalick, Bell y Schwartz 2002; Lederman y Lederman 2014). Esta conceptualización se basa en siete aspectos generales de las características de $\mathrm{NdC}$, de los cuales, seis de ellos son de perfil epistémico.

Los aspectos de la $\mathrm{NdC}$ más investigados en EP por los proponentes de esta conceptualización son (Cofré, Núñez, Santibáñez, Pavez, Valencia y Vergara 2019): (i) naturaleza provisional (la ciencia está sujeta a continuas revisiones y cambios); (ii) empirismo (la ciencia se construye a partir de evidencias del mundo natural); (iii) observaciones e inferencias (la ciencia se desarrolla a partir de observaciones del mundo natural e inferencias acerca de los fenómenos objeto de estudio); e (iv) imaginación y creatividad (la ciencia involucra la imaginación y la creatividad humana). Empleando esta conceptualización, numerosos estudios argumentan que estos aspectos de la $\mathrm{NdC}$ pueden ser comprendidos, empleando un enfoque de enseñanza explícito-reflexivo, inclusive por estudiantes del nivel elemental del sistema educativo (para dos revisiones de la literatura exhaustivas a este respecto, 
véase Akerson, Carter, Pongsanon y Nargund-Joshi 2019 y Cofré et al. 2019).

No obstante, en los últimos años han surgido perspectivas alternativas a la visión de consenso, existiendo actualmente un debate continuo acerca de qué aspectos de la $\mathrm{NdC}$ son educativamente relevantes y accesibles para los estudiantes. Así, numerosas voces críticas señalan que esta conceptualización es limitada y solo aborda las características del conocimiento científico, obviando los procesos científicos (Matthews, 2012), o que ofrece una imagen de la $\mathrm{NdC}$ demasiado simplificada y filosóficamente ingenua, resultando poco útil para entender la naturaleza de las investigaciones contemporáneas (Hodson y Wong 2017). Asimismo, otros autores subrayan que la visión de consenso de Lederman y Ledeman (2014) es demasiado restringida e incompleta (Acevedo-Díaz, García-Carmona y Aragón 2017), argumentando la necesidad de también abordar aspectos no epistémicos de la $\mathrm{NdC}$, referidos a los factores internos ( $\not$. ej., el papel de la comunicación científica o la personalidad del científico) y externos a la comunidad científica ( $\not . e j$., la influencia de la política en la ciencia o la relación entre ciencia y religión).

En el presente estudio se adopta la visión de consenso de la $\mathrm{NdC}$ propuesta por Lederman y Lederman (2014), por varios motivos. En primer lugar, para comprobar en qué medida algunos aspectos epistémicos de esta conceptualización, ampliamente promovidos e investigados en la etapa de EP, son útiles para detectar si la comprensión de la $\mathrm{NdC}$ está relacionada con una mayor valoración de la ciencia como un elemento cultural clave. En segundo lugar, porque existe una amplia bibliografía internacional que sugiere que los estudiantes de EP están preparados cognitivamente para comprender estas características epistémicas de la NdC (Akerson et al. 2019; Cofré et al. 2019); por tanto, la visión de consenso de Lederman y Lederman (2014) parecería ser una conceptualización de la NdC apropiada para la muestra de este estudio. Y, en tercer lugar, debido a que aún no existe bibliografía científica acerca de si los estudiantes de EP pueden comprender los aspectos no-epistémicos propuestos por García-Carmona y Acevedo-Díaz (2018), que sí han sido implementados eficazmente con estudiantes de Educación Secundaria Obligatoria (ESO) y Bachillerato (e.g., García-Carmona, Acevedo-Díaz y Aragón 2017).

\section{Valoración de la ciencia y actitudes hacia la implicación social de la ciencia}

El constructo de las actitudes posee una naturaleza multidimensional (Khine 2015), abarcando aspectos cognitivos (e.g., pensamientos y creencias), afectivos (e.g., sentimientos y emociones) y conductuales (e.g., acciones e intenciones). Así, las «actitudes hacia la ciencia» engloban aquellas evaluaciones individuales que se forman a medida que los individuos desarrollan creencias, emociones y experiencias sobre un objeto científico determinado (e.g., los científicos o las clases de ciencia). Por ello, este estudio posiciona la apreciación/valoración de la ciencia a la que hace referencia el argumento cultural de Driver et al. (1996) dentro del constructo de las actitudes hacia la ciencia; se considera la apreciación/valoración de la ciencia como una dimensión cognitiva de las actitudes, y se hace operativa adoptando la taxonomía de Klopfer (1971), quien desarrolla la dimensión «implicación social de la ciencia» como aquellas actitudes hacia los beneficios sociales y los problemas relacionados con la ciencia y el progreso científico. De este modo, para comprobar en qué medida los estudiantes con visiones adecuadas de la $\mathrm{NdC}$ muestran una mayor apreciación por la ciencia, se examinan las actitudes de los estudiantes hacia la dimensión actitudinal «implicación social de la ciencia», relativa a la aportación social de la ciencia y su valor para la sociedad. 


\section{Revisión de la literatura}

\section{Naturaleza de la Ciencia en Educación Primaria}

La investigación didáctica internacional en el ámbito de la EP indica que los estudiantes poseen concepciones ingenuas sobre los aspectos epistémicos de la $\mathrm{NdC}$ (Akerson y Donnelly 2010; Cakici y Bayir 2012), no existiendo diferencias en función de la variable sexo o edad (Kang, Scharmann y Noh 2005). Además, estos resultados son similares en diferentes países y culturas como, por ejemplo, en estudiantes coreanos (Yoon, Suh y Park 2014) o afroamericanos (Walls 2012). Asimismo, sugiere que los estudiantes de esta etapa son capaces de comprender estos aspectos epistémicos si se emplean enfoques didácticos explícitoreflexivos (Akerson et al. 2019).

En España, a excepción del estudio de Toma, Greca y Gómez-Orozco (2019), cuyos resultados revelan visiones ingenuas sobre la naturaleza provisional del conocimiento científico, y mixtas (esto es, ingenuas con tendencia a visiones apropiadas) en cuanto a la naturaleza empírica de la ciencia, existe un vacío de estudios que aborden la $\mathrm{NdC}$ en esta etapa educativa. Así, la investigación sobre $\mathrm{NdC}$, en este país, se ha desarrollado principalmente con estudiantes de la etapa de ESO y posteriores. En general, los resultados de estos estudios son más alentadores que los de la bibliografía educativa internacional, observándose que los estudiantes españoles de niveles post-obligatorios poseen una comprensión razonablemente adecuada de los aspectos epistémicos de la $\mathrm{NdC}$, pero con visiones más ingenuas en algunos aspectos no-epistémicos de la $\mathrm{NdC}$ relacionados con la sociología de la ciencia (VázquezAlonso, García-Carmona, Manassero-Mas y Bennásar-Roig 2013). Asimismo, García-Carmona et al. (2018) señalan que los estudiantes desde $4^{\circ}$ de ESO hasta $2^{\circ}$ de Bachillerato son capaces de mejorar su comprensión de la influencia de algunos aspectos sociológicos internos y externos en el desarrollo de la ciencia, a partir de la reflexión y discusión crítica de diferentes casos de historia de la ciencia.

\section{Actitudes hacia la ciencia en Educación Primaria}

Resulta difícil extraer conclusiones generalizables sobre las actitudes de los estudiantes de EP debido a la disparidad de constructos que han sido estudiados como reflejo de las actitudes hacia la ciencia, así como a la gran variedad de objetos de actitud examinados. Mientras que algunos estudios han explorado las actitudes hacia el «disfrute» o la «dificultad» de las clases de ciencias (Toma y Meneses-Villagrá 2019), otros han abordado aspectos diferentes como las actitudes hacia «los científicos» o hacia las «actividades científicas extracurriculares» (Navarro, Förster, González y González-Pose 2016). Por tanto, en consonancia con los objetivos de este estudio, en esta sección se revisa únicamente la bibliografía cuyo objeto actitudinal es la ciencia como una disciplina, especialmente en cuanto a su importancia y valor para la sociedad.

De este modo, estudios realizados en el contexto español con estudiantes de EP revelan que las actitudes hacia la implicación social de la ciencia tienden a ser positivas, que se mantienen a lo largo de esta etapa educativa, o inclusive mejoran en los cursos superiores (Toma et al. 2019), y que no existen diferencias significativas entre las niñas y los niños (Toma y Greca 2018; Toma, Ortiz-Revilla y Greca 2018). Otros estudios realizados con alumnado español, revelan que en la etapa de ESO la percepción de los estudiantes sobre la ciencia es más bien intermedia y poco definida (Vázquez y Manassero 2004, 2009a), con cierto decaimiento en las actitudes positivas a medida que aumenta la edad de los estudiantes; especialmente al comparar entre estudiantes de EP y ESO (Vázquez y Manassero 2008). Estos resultados contrastan con investigaciones recientes en las que predominan las visiones positivas frente a las negativas en relación con la contribución de la ciencia en la salud y el estilo de vida de las personas (de Pro y Pérez 2014). 


\section{Metodología}

\section{Diseño, participantes y procedimiento}

Se trata de un estudio cuantitativo, no experimental y correlacional (en la medida en que se estudia la relación entre las concepciones de aspectos epistémicos de la $\mathrm{NdC}$ y las actitudes hacia la implicación social de la ciencia) para el que se ha conformado un muestreo noprobabilístico intencional a partir de 7 centros educativos ubicados en la ciudad de Burgos (España), que han aceptado participar en este estudio. Cinco centros eran de adscripción concertada y dos públicos. La muestra ha estado formada por un total de 341 estudiantes matriculados en el $6^{\circ}$ curso de EP, de los cuales 156 (45.7\%) eran chicas.

\section{Instrumentos}

\section{Nature of Science Instrument (NOSI)}

Se ha empleado el instrumento de Hacıeminoğlu, Yılmaz-Tüzün y Ertepınar (2014), que examina las concepciones de los estudiantes de $6^{\circ}$ de EP a $2^{\circ}$ de ESO, en relación con cuatro características epistémicas de la $\mathrm{NdC}$ : «observaciones e inferencias», «imaginación y creatividad», «empirismo», y «naturaleza provisional». El instrumento original está compuesto por 13 ítems con tres opciones de respuestas «Falso», «No lo sé» y «Verdadero», calificados con 0,1 y 2 puntos, y viceversa en el caso de los ítems redactados de forma negativa. Tras realizar una evaluación psicométrica del instrumento con la muestra de esta investigación, se ha mantenido para su análisis posterior únicamente 9 de los 13 ítems originales, descartándose el aspecto «empirismo» (véase Anexo).

\section{Test of Science Related Attitudes (TOSRA)}

Para examinar la valoración de la ciencia, enmarcada en la dimensión actitudinal «implicación social de la ciencia», se ha empleado una escala de la versión en castellano del instrumento TOSRA, inicialmente desarrollado y validado por Fraser (1981), y recientemente sometido a una adaptación y validación cultural por Navarro et al. (2016). El instrumento original consta de siete escalas de diez ítems cada una, que miden las dimensiones de actitudes hacia la ciencia postuladas en la taxonomía de Klopfer (1971). En este estudio se usó solo la primera escala del instrumento, referida a la «implicación social de la ciencia», que mide la actitud hacia los efectos positivos o negativos de la ciencia en la sociedad, siendo, por tanto, un instrumento apropiado para el objetivo formulado en este estudio. Tras realizar una evaluación psicométrica de esta dimensión con la muestra de esta investigación, se han descartado dos ítems de los 10 originales que componen la escala, por motivos de validez y fiabilidad (véase Anexo).

No se han empleado otros instrumentos desarrollados en la bibliografía española por los siguientes motivos: de Pro y Pérez (2014) han propuesto un instrumento para medir actitudes ante la visión dicotómica de la ciencia (efectos positivos y negativos), sin embargo, no han reportado datos sobre su validez y fiabilidad. Asimismo, Vázquez y Manassero (2008) han empleado el instrumento ROSE, cuya evaluación psicométrica revela una estructura factorial inconsistente (ítems con cargas cruzadas en varios factores, i.e., Vázquez y Manassero 2004) y poca fiabilidad (Alfa de Cronbach $=0.250$ para el factor que mide la «imagen social de la ciencia y la tecnología», i.e., Vázquez y Manassero 2009b). Finalmente, la escala «progreso social» de Vázquez y Manassero (2004), que mide aspectos similares a la empleada en este estudio, está compuesta por ítems que hacen referencia tanto a la ciencia como a la tecnología (e.g., "3. La ciencia y la tecnología hacen nuestra vida más saludable, más fácil y más cómoda”, p. 389, énfasis añadido), siendo la tecnología un aspecto ajeno al alcance de este estudio y, además, tratándose de ítems de doble calibre que combinan dos objetos actitudinales distintos (ciencia 
y tecnología) y dos características de estas disciplinas (más fácil y más cómoda) en una sola puntuación, afectando la exactitud y fiabilidad de los resultados obtenidos (DeVellis 2017).

\section{Procedimiento y análisis de datos}

Los instrumentos fueron administrados por el autor de este estudio, en formato papel, al comienzo del tercer trimestre del curso académico 2018-19, empleando en cada centro escolar una clase lectiva de la asignatura Ciencias de la Naturaleza. A fin de evitar respuestas sesgadas por deseabilidad social, se informó a los estudiantes del carácter anónimo, voluntario y confidencial de su participación.

Para identificar las concepciones sobre la $\mathrm{NdC}$, las respuestas equivocadas o marcadas como «No lo sé» fueron categorizadas como reflejo de una visión ingenua o poco apropiada de la $\mathrm{NdC}$, y aquellas correctas como una visión apropiada. Posteriormente, se sumaron las respuestas de los ítems de cada dimensión, considerándose que el estudiante posee una visión apropiada de cada aspecto epistémico de la $\mathrm{NdC}$ en los casos cuyas respuestas fueron correctas en al menos un 75\% de los ítems, lo que corresponde a 3 o 4 respuestas adecuadas para el aspecto «creatividad e imaginación», 3 respuestas adecuadas para «naturaleza provisional», y 2 respuestas adecuadas para «observación e inferencias» ${ }^{1}$. Por otro lado, para determinar las actitudes de los estudiantes hacia la implicación social de la ciencia, se sumaron las respuestas de cada ítem y se calculó la media para cada estudiante, considerando una puntuación inferior a 2.5 puntos, en una escala de 4 posibles opciones de respuesta (del 1 al 4 , «Totalmente en desacuerdo» «En desacuerdo», «De acuerdo» y «Totalmente de acuerdo»), como reflejo de una actitud negativa.

Para examinar las actitudes y la comprensión de la NdC de los estudiantes, se empleó análisis estadístico descriptivo, así como comparaciones según la variable sexo de los estudiantes empleando análisis estadístico inferencial no paramétrico (prueba $\chi^{2}$ de Pearson) para cada instrumento y dimensión, debido a la violación de la asunción de distribución normal, según la prueba Kolmogorov-Smirnov $(\phi<0.001)$. Para establecer la relación entre la comprensión de los aspectos epistémicos de la naturaleza de la ciencia promovidos en EP por los proponentes de la visión de consenso de Lederman y Lederman (2014) y las actitudes hacia la implicación social de la ciencia, se realizaron tres pruebas $U$ de Mann-Whitney, que comparan las puntuaciones de actitud hacia la implicación social de la ciencia de los estudiantes (variable dependiente) en función de una visión ingenua o apropiada de las tres dimensiones epistémicas de la $\mathrm{NdC}$ estudiadas (variable de agrupación). Se aplicó la corrección de Bonferroni para ajustar el nivel de significancia a un valor más restrictivo $(\phi<0.01)$ y así reducir la probabilidad de cometer errores de tipo I, producto de pruebas inferenciales repetidas. La importancia educativa de los resultados se determinó en base a los criterios de Cohen (1988) para el tamaño del efecto, según el cual 0.1 se considera pequeño, 0.3 , moderado y 0.5 , grande.

\section{Resultados}

\section{Comprensión de aspectos epistémicos de la $\mathrm{NdC}$}

En general, los estudiantes de esta muestra poseen una comprensión ingenua de los aspectos epistémicos de la $\mathrm{NdC}$ examinados en este estudio (véase la Figura 1). Más específicamente, el $80.6 \%$ de los estudiantes consideran que el conocimiento científico no está influenciado por la creatividad e imaginación de los científicos, el 85.9\% concibe el conocimiento científico como

\footnotetext{
${ }_{1}^{1}$ Hacieminoğlu et al. (2014) otorgan 2 puntos para las respuestas correctas y 1 punto para aquellas marcadas como «No lo sé». Dado que la comprensión de la $\mathrm{NdC}$ es un aspecto cognitivo, esta forma de evaluar las ítems introduce sesgo sobre el verdadero conocimiento que poseen los estudiantes en relación con los aspectos epistémicos de la $\mathrm{NdC}$, pues valora positivamente aquellas respuestas que el estudiante no conoce.
} 
una verdad absoluta que no está sujeta a revisiones o modificaciones, y el 77.1\% no considera que el conocimiento científico se desarrolle a partir de observaciones e inferencias.

La prueba de independencia Chi-cuadrado indica que no existe una asociación estadísticamente significativa entre la variable de sexo y los aspectos «creatividad e imaginación», $\chi^{2}(1, n=341)=0.003, p=0.54, \mathrm{y}$ «naturaleza provisional» de la $\mathrm{NdC}, \chi^{2}(1, n$ $=341)=1.531, p=0.14$. No obstante, se identificó una mayor proporción de chicos $(69.2 \%)$ con visiones apropiadas en el aspecto «observaciones e inferencias» que de chicas (30.8\%), aunque con un tamaño de efecto muy bajo, por lo que este resultado carece de importancia educativa, $\chi^{2}(1, n=341)=9.142, p<0.01$, phi $=0.02$.

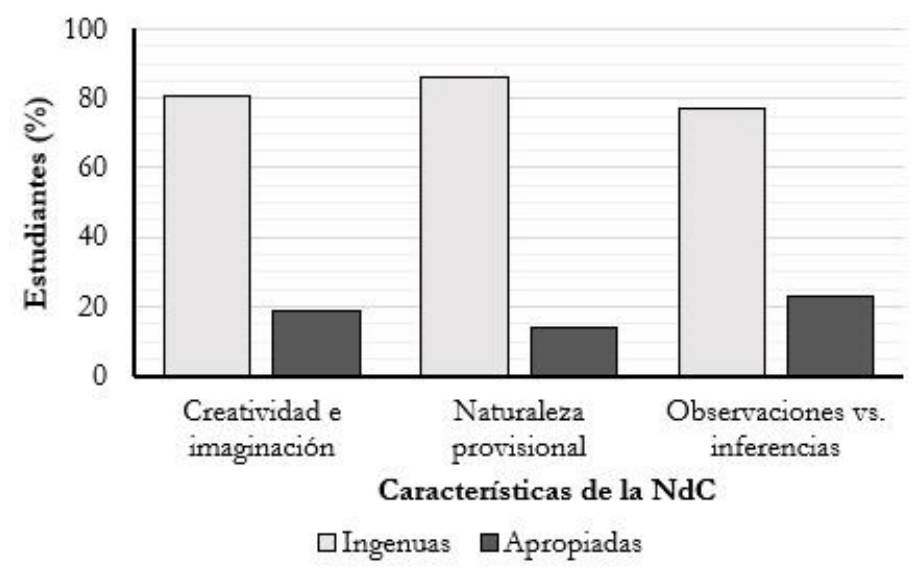

Figura 1. Concepciones sobre aspectos epistémicos de la NdC.

\section{Actitudes hacia la implicación social de la ciencia}

Los estudiantes de esta muestra han reportado una actitud positiva hacia la implicación social de la ciencia, con una media de $3.46(D E=0.65)$ de 4 posibles puntos. La prueba $U$ de MannWhitney revela que existe una diferencia estadísticamente significativa entre la puntuación de las chicas $(M d=3.5 ; M=3.25 ; D E=0.74)$ y la de los chicos $(M d=4 ; M=3.64 ; D E=0.50)$, $U=9840, z=-5.38, p<0.01, r=0.30$. Estos resultados sugieren que los chicos poseen una actitud más positiva hacia la implicación social de la ciencia que las chicas, con un tamaño de efecto moderado. No obstante, cabe destacar que, en términos generales, ambos grupos poseen unas actitudes positivas.

Al agrupar las respuestas en categorías negativas y positivas, considerando una puntuación inferior a 2.5 puntos como reflejo de una actitud negativa, un $84.5 \%$ de los estudiantes de esta muestra han reportado una actitud positiva hacia la implicación social de la ciencia. La prueba de independencia Chi-cuadrado proporciona una asociación estadísticamente significativa entre las actitudes y la variable sexo, $\chi^{2}(1, n=341)=12.435, p<0.01$, phi $=0.19$. Los resultados indicaron que la proporción de chicas con actitudes negativas hacia la implicación social de la ciencia es dos veces mayor que la de los chicos (véase la Figura 2). 


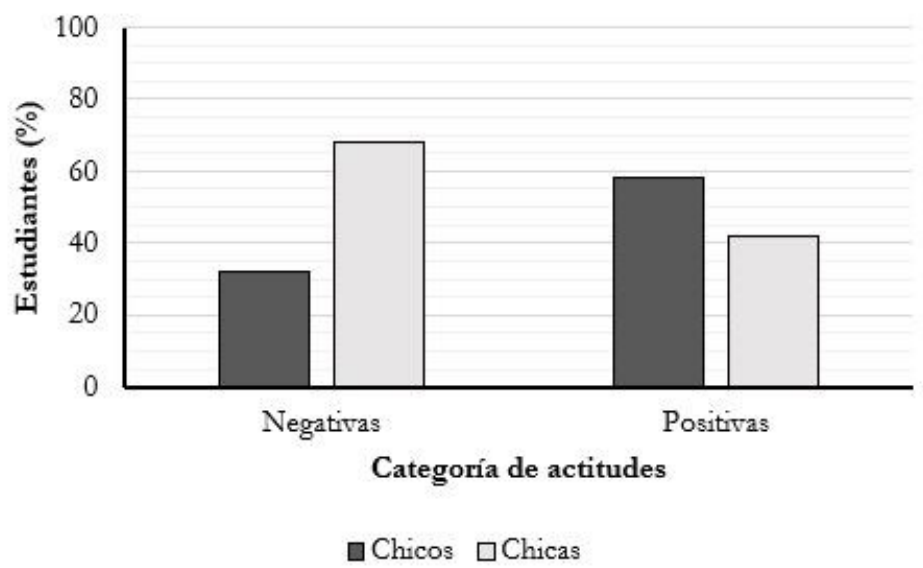

Figura 2. Actitudes hacia la implicación social de la ciencia según sexo.

\section{Actitudes hacia la implicación social de la ciencia según comprensión de la NdC}

En general, los resultados sugieren una relación parcial entre la comprensión de aspectos epistémicos de la $\mathrm{NdC}$ y actitudes hacia la implicación social de la ciencia (véase la Tabla 1). Más específicamente, por un lado, no hubo diferencias estadísticamente significativas entre las actitudes de los estudiantes con una comprensión ingenua y aquellos con una comprensión adecuada del aspecto epistémico «creatividad e imaginación» de la $\mathrm{NdC}, U=7842$,,$=-1.82$, $p=0.07$. No obstante, por otro lado, sí hubo diferencias estadísticamente significativas para los aspectos «observaciones e inferencias», $U=7233, z=-4.20, p<0.01, r=0.30, \mathrm{y}$ «naturaleza provisional», $U=5073, z=-3.29, p<0.01, r=0.51$, en las que los estudiantes con una comprensión más adecuada de estos aspectos epistémicos de la $\mathrm{NdC}$ reportaron unas actitudes más positivas hacia la implicación social de la ciencia, con medio y gran tamaño de efecto, respectivamente. Estos resultados sugieren que los estudiantes que poseen una comprensión adecuada de la naturaleza provisional de la ciencia y que comprenden que el conocimiento científico se construye a partir de observaciones e inferencias, poseen una puntuación más elevada en la dimensión actitudinal de implicación social de la ciencia y, por ende, una mayor apreciación de la ciencia que sus pares con visiones ingenuas.

Tabla 1. Resultados descriptivos para la «implicación social de la ciencia», según comprensión de aspectos epistémicos de la NdC.

\begin{tabular}{|c|c|c|c|c|c|c|}
\hline \multirow{2}{*}{ Descriptivos } & \multicolumn{2}{|c|}{ Creatividad e imaginación } & \multicolumn{2}{|c|}{ Naturaleza provisional } & \multicolumn{2}{|c|}{$\begin{array}{c}\text { Observaciones e } \\
\text { inferencias }\end{array}$} \\
\hline & Ingenuas & Apropiadas & Ingenuas & Apropiadas & Ingenuas & Apropiadas \\
\hline$M$ & $\begin{array}{c}3.43 \\
(3.35-3.51)\end{array}$ & $\begin{array}{c}3.59 \\
(3.45-3.73)\end{array}$ & $\begin{array}{c}3.42 \\
(3.35-3.50)\end{array}$ & $\begin{array}{c}3.68^{*} \\
(3.52-3.85)\end{array}$ & $\begin{array}{c}3.38 \\
(3.30-3.46)\end{array}$ & $\begin{array}{c}3.73 * \\
(3.64-3.83)\end{array}$ \\
\hline$D E$ & 0.67 & 0.56 & 0.67 & 0.56 & 0.68 & 0.42 \\
\hline$M d$ & 3.5 & 4 & 3.5 & 4 & 3.5 & 4 \\
\hline
\end{tabular}

\section{Discusión de los resultados}

El propósito de esta investigación fue examinar en qué medida una concepción adecuada sobre algunos aspectos epistémicos de la $\mathrm{NdC}$ está asociada con una mayor apreciación de la ciencia; la cual se ha concretado en una valoración de las actitudes hacia la implicación social de la ciencia y su papel en la sociedad. En conjunto, los resultados de este estudio ofrecen un apoyo empírico, aunque parcial, al argumento cultural de Driver et al. (1996) para la enseñanza de 
la $\mathrm{NdC}$ en $6^{\circ}$ de EP. Mientras que los estudiantes con concepciones informadas sobre los aspectos «observaciones e inferencias» $\mathrm{y}$ «naturaleza provisional» de la ciencia reportaron actitudes más positivas que sus pares con visiones ingenuas, no hubo diferencias en sus actitudes en función del aspecto epistémico denominado «creatividad e imaginación».

Estos hallazgos, que sugieren que una comprensión adecuada de aspectos epistémicos de la $\mathrm{NdC}$ podría estar relacionada con actitudes de valoración de los beneficios sociales de la ciencia, contrastan con estudios previos que han examinado otros supuestos teóricos existentes con respecto a la aportación educativa de los aspectos epistémicos de la $\mathrm{NdC}$. Por ejemplo, Bell y Lederman (2002) concluyeron que la toma de decisiones en cuestiones científico-tecnológicas no está influida por la comprensión de la NdC. El estudio de Khishfe (2012) tampoco reveló ningún impacto de la comprensión de la NdC en la toma de decisiones por parte de los estudiantes en cuestiones socio-científicas relacionadas con alimentos genéticamente modificados. El estudio de Sinatra, Southerland, McConaughy y Demastes (2003) mostró igualmente que no había relación entre las creencias epistemológicas de los estudiantes y su aceptación de la teoría de la evolución o la teoría de la fotosíntesis. Asimismo, en un estudio con profesorado de biología chilenos, no se encontró ninguna relación entre la comprensión de la $\mathrm{NdC}$ y la comprensión y aceptación de la teoría de la evolución (Cofré et al. 2017). No obstante, cabe remarcar que todos estos estudios, así como el presente, han adoptado la visión de consenso de la NdC (Lederman y Lederman, 2014), que se limita esencialmente a aspectos epistémicos de la $\mathrm{NdC}$; por tanto, una visión más holística de la $\mathrm{NdC}$, que incluya aspectos no-epistémicos (Acevedo-Díaz et al. 2017; García-Carmona et al. 2018), podría dar lugar a resultados diferentes.

Por otro lado, el análisis de las respuestas a las escalas de $\mathrm{NdC}$ y actitudes revela que la mayoría de los estudiantes del $6^{\circ}$ curso de EP incluidos en este estudio poseen, por un lado, una concepción ingenua de los aspectos epistémicos de la $\mathrm{NdC}$ analizados (en este caso, creatividad e imaginación, naturaleza provisional, y observaciones e inferencias), y por otro, actitudes positivas hacia la implicación social de la ciencia. Estos resultados son consistentes con estudios internacionales (Yoon et al. 2014) y nacionales (Toma et al. 2019) sobre NdC, así como con la bibliografía sobre actitudes con estudiantes de EP (e.g., de Pro y Pérez 2014, Toma y Meneses Villagrá 2019b).

\section{Conclusiones y prospectiva}

Hace más de dos décadas, Driver et al. (1996) formularon cinco argumentos a favor de la enseñanza de la $\mathrm{NdC}$ en el sistema educativo. Esta investigación ofrece apoyo empírico parcial a uno de ellos, denominado el argumento cultural, que postula que una comprensión adecuada de la $\mathrm{NdC}$ es necesaria para que los ciudadanos puedan apreciar la ciencia como un elemento importante de la cultura contemporánea. Para Driver et al. (1996), los ciudadanos deben tener una comprensión epistemológica adecuada para que sean capaces de entender “(...) las elegantes y poderosas estructuras de las ideas que nosotros hemos desarrollado para la comprensión de los fenómenos y eventos naturales" (p. 19). En palabras de los autores, una "apreciación de la ciencia "(...) requeriría inevitablemente la comprensión de las cuestiones e ideas epistemológicas (...)” (p. 20).

Si bien los resultados de este estudio permiten apoyar el argumento cultural para la enseñanza de $\mathrm{NdC}$ en $6^{\circ}$ curso de EP, es posible que la adición de los aspectos no-epistémicos de la misma (Acevedo-Díaz et al. 2017; García-Carmona et al. 2018), no abordados en esta investigación, contribuyan a fortalecer la relación entre las concepciones epistemológicas de los estudiantes y el desarrollo de sus actitudes hacia la implicación social de la ciencia. Por tanto, se demanda la realización de futuros estudios que aborden esta temática adoptando un marco de la $\mathrm{NdC}$ más 
amplio que el de la visión de consenso, el cual ya ha sido señalado como una perspectiva limitada y restringida de la NdC (García-Carmona et al. 2018; Hodson y Wong 2017).

Finalmente, se espera que este estudio promueva futuras investigaciones que exploren cómo la comprensión de la $\mathrm{NdC}$ puede contribuir al desarrollo de actitudes de valoración de la ciencia en los estudiantes. La respuesta a ello tendría una implicación directa en el desarrollo del currículo de ciencias; es decir, si futuras investigaciones confirman los resultados del presente estudio, la inclusión de la $\mathrm{NdC}$ en los currículos de ciencias se tornaría aún más crítica y necesaria.

\section{Agradecimientos}

Agradezco al editor de sección y a los evaluadores anónimos por revisar críticamente el manuscrito y proporcionar comentarios constructivos.

\section{Referencias}

Acevedo-Díaz, J. A. (2009) Enfoques explícitos versus implícitos en la enseñanza de la naturaleza de la ciencia. Revista Eureka sobre Enseñanza y Divulgación de las Ciencias, 6(3), 355386.

Acevedo-Díaz, J. A., García-Carmona, A., Aragón, M. M. (2017) Enseñar y aprender sobre naturaleza de la Ciencia mediante el análisis de controversias de historia de la Ciencia: Resultados y conclusiones de un proyecto de investigación didáctica. Madrid: Organización de Estados Iberoamericanos para la Educación, la Ciencia y la Cultura (OEI).

Akerson, V. L., Carter, I., Pongsanon, K., Nargund-Joshi, V. (2019) Teaching and learning nature of science in elementary classrooms. Research-based strategies for practical implementation. Science \& Education, 28, 391-311. https://doi.org/10.1007/s11191-01900045-1

Akerson, V. L., Donnelly, L. A. (2010) Teaching nature of science to K-2 students: What understandings can they attain? International Journal of Science Education, 32(1), 97-124. https://doi.org/10.1080/09500690902717283

Bell, R. L., Lederman, N. G. (2003) Understandings of the nature of science and decision making on science and technology based issues. Science Education, 87(3), 352-377.

Cakici, Y., Bayir, E. (2012) Developing children's views of nature of science through role play. International Journal of Science Education, 34(7), 1075-1091. https://doi.org/10.1080/09500693.2011.647109

Cohen, J. (1988) Statistical power analysis for the behavioral sciences. Hillsdale: Lawrence Erlbaum Associates.

Cofré, H., Cuevas, E., Becerra, B. (2017) The relationship between biology teachers' understanding of the nature of science and the understanding and acceptance of the theory of evolution. International Journal of Science Education, 39(16), 2243-2260. https://doi.org/10.1080/09500693.2017.1373410

Cofré, H., Nuñez, P., Santibáñez, D., Pavez, J. M., Valencia, M., Vergara, C. (2019) A critical review of students' and teachers' understandings of nature of science. Science \& Education, online first 1-44. https://doi.org/10.1007/s11191-019-00051-3

de Pro Bueno, A., Pérez, M. A. (2014) Actitudes de los alumnos de Primaria y Secundaria ante la visión dicotómica de la Ciencia. Enseñanza de las Ciencias, 32(3), 111-132. https://doi.org/10.5565/rev/ensCiencias.1015 
DeVellis, R. F. (2017) Scale development. Theory and applications. Los Angeles: SAGE.

Driver, R., Leach, J., Millar, R., Scott, P. (1996) Young people's images of science. Philadelphia: Open University Press.

Evans, G., Durant, J. (1995) The relationship between knowledge and attitudes in the public understanding of science in Britain. Public Understanding of Science, 4. 57-74.

Fraser, B. (1981) Test of Science Related Attitudes. Melbourne: Australian Council for Educational Research

García-Carmona, A., Acevedo-Díaz, J. A., Aragón M. M. (2018) Comprensión del alumnado de Secundaria sobre la dimensión sociológica de la naturaleza de la Ciencia a partir de la historia de la Ciencia. Ápice. Revista de Educación Científica, 2(2), 43-54. https://doi.org/10.17979/arec.2018.2.2.4519

Hacıeminoğlu, E., Yılmaz-Tüzün, Ö., Ertepınar, H. (2014) Development and validation of nature of science instrument for elementary school students. International Journal of Primary, Elementary and Early Years Education, 42(3), 258-283. https://doi.org/10.1080/03004279.2012.671840

Harty, H., Samuel, J. V., Andersen, H. O. (1991) Understanding the nature of science and attitudes toward science and science teaching of preservice elementary teachers in three preparation sequences. Journal of Elementary Science Education, 3(1), 12-22.

Hodson, D., Wong, S. L. (2017) Going beyond the consensus view: Broadening and enriching the scope of NOS oriented curricula. Canadian Journal of Science, Mathematics and Technology Education, 17(1), 3-17. https://doi.org/10.1080/14926156.2016.1271919

Kang, S., Scharmann, L. C., Noh, T. (2005) Examining students' views on the nature of science: Results from Korean $6^{\text {th }}, 8^{\text {th }}$, and $10^{\text {th }}$ graders. Science Education, 89(2), 314-334.

Khine, M. S. (2015) Attitude measurements in science education: Classic and contemporary approaches. Charlotte, N. C.: Information Age Publishing, INC.

Khishfe, R. (2012) Nature of Science and decision-making. International Journal of Science Education, 34(1), 67-100. https://doi.org/10.1080/09500693.2011.559490

Klopfer, L. E. (1971) Evaluation of learning in science. En B. S. Bloom, J. T. Hastings, y G. F. Madaus (Eds.), Handbook of formative and summative evaluation of student learning. London: McGraw-Hill.

Lederman, N. G., Abd-El-Khalick, F., Bell, R. L., Schwartz, R. S. (2002) Views of nature of science questionnaire: Toward valid and meaningful assessment of learners' conceptions of nature of science. Journal of Research in Science Teaching, 36(6), 497-521. https://doi.org/10.1002/tea.10034

Lederman, N. G. (2007) Nature of science: Past, present, and future. En S. K. Abell y N. G. Lederman (Eds.), Handbook of research on science education (Vol. I, pp. 831-879). Mahwah: Lawrence Erlbaum Publishers.

Lederman, N. G., Bartos, S. A., Lederman, J. S. (2014) The development, use, and interpretation of nature of science assessments. En M. R. Matthews (ed.), International Handbook of Research in History, Philosophy and Science Teaching, (pp. 971-997). Dordrecht: Springer. 
Lederman, N. G., Lederman, J. S. (2014) Research on teaching and learning of nature of science. En N. G. Lederman y S. K. Abell (Eds.), Handbook of research on science education (Vol. II, pp. 600-620). New York: Routledge.

Lederman, N. G. (1999) Teachers' understanding of the nature of science and classroom practice: Factors that facilitate or impede the relationship. Journal of Research in Science Teaching, 36(8), 919-929.

McComas, W. F. (2017) Understanding how science works: The nature of science as the foundation for science teaching and learning. School Science Review, 98(365), 71-76.

Navarro, M., Förster, C., González, C., González-Pose, P. (2016) Attitudes toward science: Measurement and psychometric properties of the Test of Science-Related Attitudes for its use in Spanish-speaking classrooms. International Journal of Science Education, 38(9), 14591482. https://doi.org/10.1080/09500693.2016.1195521

Nunnally, J. C., Bernstein, I. H. (1994) Psychometric theory. New York: McGraw-Hill.

Olson, J. K. (2018) The inclusion of the nature of science in nine recent international science education standards documents. Science \& Education, 27(7-8), 637-660. https://doi.org/10.1007/s11191-018-9993-8

Sinatra, G. M., Southerland, S. A., McConaughy, F., Demastes, J. W. (2003) Intentions and beliefs in students' understanding and acceptance of biological evolution. Journal of Research in Science Teaching, 40(5), 510-528. https://doi.org/10.1002/tea.10087

Tabachnick, B. G., Fidell, L. S. (2007) Using multivariate statistics (5 ${ }^{\text {th }}$ ed.). Boston, MA: Allyn \& Bacon/Pearson Education.

Toma, R. B., Greca, I. M. (2018) The effect of integrative STEM instruction on elementary students' attitudes toward science. Eurasia Journal of Mathematics, Science and Technology Education, 14(4), 1383-1395. https://doi.org/10.29333/ejmste/83676

Toma, R. B., Greca, I. M., Orozco-Gómez, M. L. (2019) Attitudes towards science and views of nature of science among elementary school students in terms of gender, cultural background and grade level variables, Research in Science \& Technological Education, online first 1-24, https://doi.org/10.1080/02635143.2018.1561433

Toma, R. B., Meneses Villagrá, J. A. (2019a) Preferencia por contenidos científicos de física o de biología en Educación Primaria: Un análisis clúster. Revista Eureka sobre Enseñanża y Divulgación de las Ciencias, 16(1), 1104. https://doi.org/10.25267/Rev_Eureka_ensen_divulg_cienc.2019.v16.11.1104

Toma, R. B., Meneses Villagrá, J. A. (2019b) Validation of the single-items Spanish-School Science attitude survey (S-SSAS) for elementary education. PloS ONE, 14(1), e0209027. https://doi.org/10.1371/journal.pone.0209027

Toma, R. B., Ortiz-Revilla, J., Greca, I. M. (2018) Actitudes hacia la ciencia de alumnado interesado en actividades científicas extracurriculares. En C. Martínez Losada y S. García Barros (eds.), $28^{\circ}$ Encuentros de Didáctica de las Ciencias Experimentales. Iluminando el cambio educativo. A Coruña: Universidade da Coruña.

Vázquez, A., Manassero, M. A. (2004) Imagen de la ciencia y la tecnología al final de la educación obligatoria. Cultura y Educación, 16(4), 385-398.

Vázquez, A., Manassero, M. A. (2008) El declive de las actitudes hacia la Ciencia de los estudiantes: Un indicador inquietante para la educación científica. Revista Eureka Sobre Enseñanza y Divulgación de Las Ciencias, 5(3), 274-292. 
Vázquez, A., Manassero, M. A. (2009a) La relevancia de la educación científica: actitudes y valores de los estudiantes relacionados con la Ciencia y la Tecnología. Enseñanza de las Ciencias, 27(1), 33-48.

Vázquez, A., Manassero, M. A. (2009b) Factores actitudinales determinantes de la vocación científica y tecnológica en secundaria. Cultura y Educación, 21(3), 319-330.

Vázquez-Alonso, Á., García-Carmona, A., Manassero-Mas, M. A., Bennassar-Roig, A. (2013) Spanish secondary-school science teachers' beliefs about Science-Technology-Society (STS) Issues. Science \& Education, 22(5), 1191-1218. https://doi.org/10.1007/s11191-012-9440-1

Walls, L. (2012) Third grade African American students views of the nature of science. Journal of Research in Science Teaching, 49(1), 1-37. https://doi.org/10.1002/tea.20450

Yoon, S. Y., Suh, J. K., Park, S. (2014) Korean students' perceptions of scientific practices and understanding of nature of science. International Journal of Science Education, 36(16), 26662693. https://doi.org/10.1080/09500693.2014.928834 


\begin{abstract}
Anexo
Este anexo recoge la versión final de los instrumentos de recogida de datos empleados, así como su análisis psicométrico con respecto a la validez de constructo y fiabilidad, realizado con la muestra de este estudio.
\end{abstract}

\title{
Traducción del instrumento NOSI
}

El instrumento NOSI (Hacieminoğlu et al. 2014) fue traducido al castellano siguiendo un método de validación intercultural, según el cual un traductor bilingüe tradujo los ítems del inglés al español (foreward translation), y otro tradujo los ítems del español nuevamente al idioma original (back translation). Finalmente, ambos traductores y el autor de este trabajo revisaron conjuntamente la equivalencia entre la versión original y la versión traducida, adaptando algunos ítems al nivel de comprensión y conocimiento de los estudiantes españoles de Educación Primaria de esta muestra. Así, el ítem 7 fue modificado añadiendo ejemplos cercanos al contexto de la ciudad de Burgos (España), cambiando del original «Los científicos están seguros de la estructura de los átomos porque pudieron verlos a través de microscopios» (Hacieminoğlu et al. 2014, p. 269) a «Los científicos están seguros de la existencia de los dinosaurios porque pudieron encontrar sus fósiles». Este cambio fue repetido en el ítem 8, adaptado del original «Los científicos no pueden estar seguros de la estructura de los átomos porque no pudieron verlos a través del microscopio; tan solo dedujeron que existen» (p. 269) a «Los científicos no pueden estar seguros de la forma de los dinosaurios porque no pudieron verlos en la realidad; tan solo dedujeron su forma». Finalmente, el ítem 9 fue modificado del original «La teoría atómica moderna aceptada hoy podría cambiar en el futuro a medida que los científicos obtengan nuevas pruebas» (p. 269) a «Las teorías científicas aceptadas hoy podrían cambiar en el futuro a medida que los científicos obtengan nuevas pruebas». Por último, los docentes de los centros educativos que participaron en el estudio, revisaron la versión final confirmando su idoneidad para la comprensión lectora y conceptual de los estudiantes que componen la muestra de este estudio.

\section{Procedimiento para la evaluación psicométrica de los instrumentos}

Para la validez de constructo, que valora si el instrumento capta la dimensionalidad hipotética del constructo objeto de estudio, se efectuó un análisis de componentes principales, empleándose el método de extracción de componente principales con rotación Varimax. Se usó el análisis paralelo para decidir el número de factores a retener. Posteriormente, se han retenido aquellos ítems que han alcanzado un valor mínimo de 0.30 para la comunalidad y 0.45 para las saturaciones factoriales, eliminándose aquellos con saturaciones cruzadas entre dos factores superiores a 0.10 (Tabachnick y Fidell 2007). Para examinar la fiabilidad de los factores retenidos, que refiere al grado en que los ítems de un factor están interrelacionados entre sí, se empleó el índice Alfa de Cronbach con un valor de $\alpha>0.60$ como mínimo aceptable y $\alpha>0.70$ como deseable (Nunnally y Bernstein 1994). De acuerdo con los estudios de validación originales, se espera alcanzar una solución factorial de estructura simple de cuatro factores para el NOSI (Hacieminoğlu et al. 2014) y una estructura unidimensional para la dimensión «implicación social de la Ciencia» del instrumento TOSRA (Fraser 1981).

\section{Resultados}

\section{Propiedades psicométricas del instrumento NOSI}

El análisis de componentes principales reveló la existencia de cuatro factores con valores propios (eigenvalues) superiores a $\mathrm{K}>1$. No obstante, el análisis paralelo indicó que la extracción de solo tres factores proporcionaría una estructura más parsimoniosa. 
$\mathrm{Al}$ extraer tres factores, los ítems 3, 9 y 13 mostraron cargas cruzadas superiores a 0.10 y el ítem 11 tuvo una comunalidad inferior al valor mínimo de 0.30 . Tras eliminar estos cuatro ítems, se extrajo tres factores con un 58.3\% de varianza total explicada. La rotación Varimax reveló la existencia de una estructura simple en la que los ítems poseen cargas factoriales para un solo factor y sin cargas cruzadas entre los mismos (véase la Tabla 2). De este modo, el primer factor está compuesto por cuatro ítems con saturaciones medias de 0.74 que miden la dimensión de «Creatividad e Imaginación» de la $\mathrm{NdC}(\alpha=0.75)$; el segundo factor está compuesto por tres ítems con saturaciones medias de 0.73 que miden la naturaleza «provisional» de la Ciencia $(\alpha=0.66)$; finalmente, el tercer factor está compuesto por dos ítems con saturaciones medias de 0.73 enfocados a medir la dimensión «Observaciones e inferencias» de la $\mathrm{NdC}(\alpha=0.61)$.

Tabla 2. Análisis de componentes principales para el instrumento NOSI.

\begin{tabular}{|c|c|c|c|c|}
\hline \multirow{2}{*}{ Ítems } & \multicolumn{3}{|c|}{ Factores } & \multirow{2}{*}{$b^{2}$} \\
\hline & 1 & 2 & 3 & \\
\hline $\begin{array}{l}\text { 1. El conocimiento científico no cambia, porque si los científicos no están } \\
\text { seguros de ello, no lo ponen en los libros para los estudiantes (i) }\end{array}$ & -0.06 & 0.713 & 0.02 & 0.51 \\
\hline $\begin{array}{l}\text { 2. Todo lo que los científicos dicen en los libros no cambia en ningún momento } \\
\text { (i) }\end{array}$ & -0.03 & 0.75 & 0.17 & 0.58 \\
\hline $\begin{array}{l}\text { 3. El conocimiento científico puede cambiar en el sentido de añadir nuevos } \\
\text { datos para ampliar el conocimiento }\end{array}$ & - & - & - & - \\
\hline $\begin{array}{l}\text { 4. Los científicos sólo pueden añadir nuevos conocimientos, pero no pueden } \\
\text { quitar nada de lo que ya se sabe (i) }\end{array}$ & 0,13 & 0.72 & $-0,15$ & 0.54 \\
\hline 5. Los científicos usan su imaginación durante sus investigaciones & 0.81 & -0.04 & 0.07 & 0.67 \\
\hline 6. Los científicos utilizan su creatividad en sus investigaciones & 0.74 & -0.04 & 0.05 & 0.56 \\
\hline $\begin{array}{l}\text { 7. Los científicos están seguros de la existencia de los dinosaurios porque } \\
\text { pudieron encontrar sus fósiles }\end{array}$ & 0.10 & 0.11 & 0.70 & 0.53 \\
\hline $\begin{array}{l}\text { 8. Los científicos no pueden estar seguros de la forma de los dinosaurios } \\
\text { porque no pudieron verlos en la realidad; tan solo dedujeron su forma }\end{array}$ & 0.13 & 0.17 & 0.77 & 0.62 \\
\hline $\begin{array}{l}\text { 9. Las teorías científicas aceptadas hoy podrían cambiar en el futuro a medida } \\
\text { que los científicos obtengan nuevas pruebas }\end{array}$ & - & - & - & - \\
\hline $\begin{array}{l}\text { 10. La ciencia nunca podría involucrar aspectos humanos como la imaginación } \\
\text { y la creatividad porque daría lugar a resultados incorrectos (i) }\end{array}$ & 0.72 & -0.07 & 0.28 & 0.62 \\
\hline $\begin{array}{l}\text { 11. El conocimiento científico puede ser influenciado por la imaginación y la } \\
\text { creatividad de los científicos }\end{array}$ & - & - & - & - \\
\hline $\begin{array}{l}\text { 12. El conocimiento científico depende solo de los datos obtenidos en } \\
\text { experimentos, no de la imaginación y creatividad de los científicos (i) }\end{array}$ & 0.69 & 0.03 & -0.33 & 0.54 \\
\hline $\begin{array}{l}\text { 13. Los científicos pueden llegar a conclusiones diferentes cuando analizan los } \\
\text { mismos datos }\end{array}$ & - & - & - & - \\
\hline Valores propios (eigenvalues) & 2.31 & 1.63 & 1.34 & - \\
\hline $\begin{array}{l}h^{2} \text { refiere a las comunalidades de los ítems } \\
\text { (i) refiere a los ítems cuyos resultados deben ser invertidos antes del análisis } \\
\text { - refiere a los ítems que han sido eliminados para el análisis de componentes pri } \\
\text { instrumento }\end{array}$ & & & & \\
\hline
\end{tabular}

\section{Propiedades psicométricas de la dimensión «implicación social de la ciencia»}

El análisis de componentes principales reveló la existencia de dos factores con valores propios $\mathrm{K}>$ 1. Sin embargo, el análisis paralelo indicó que una extracción unidimensional proporcionaría una estructura más parsimoniosa. Al extraer un solo factor, los ítems 3 y 6 mostraron una comunalidad muy inferior al valor 0.30 . Tras eliminar estos ítems, se ha extraído un solo factor con un $35.7 \%$ de varianza total explicada (véase la Tabla 3). Este factor está compuesto por ocho ítems con saturaciones medias de 0.60 que miden las actitudes de los estudiantes hacia la implicación social de la Ciencia $(\alpha=0.72)$. Si bien el ítem 7 y 10 poseen comunalidades ligeramente inferiores al valor mínimo establecido, fueron retenidos en el factor final pues su exclusión reduce considerablemente la confiabilidad del instrumento (al excluir ítems, $\alpha=0.67$ ). 
Tabla 3. Análisis de componentes principales para la escala actitudinal «implicación social de la ciencia».

\begin{tabular}{lcc}
\hline Ítems & Factor \\
\cline { 2 - 3 } & 1 & $h^{2}$ \\
\hline 1. El dinero que se invierte en ciencias es dinero bien invertido & $\mathbf{0 . 6 0}$ & 0.36 \\
2. La ciencia es el peor enemigo del hombre (i) & $\mathbf{0 . 6 6}$ & 0.41 \\
3. El dinero público dedicado a la ciencia en los últimos años ha sido utilizado con inteligencia & - & - \\
4. Los descubrimientos científicos están produciendo más daño que beneficio (i) & $\mathbf{0 . 5 5}$ & 0.31 \\
5. El gobierno debe gastar más dinero en la investigación científica & $\mathbf{0 . 7 1}$ & 0.50 \\
6. Se están construyendo demasiados laboratorios a costa de la disminución de inversión para el resto de la & - & - \\
educación (i) & $\mathbf{0 . 5 1}$ & 0.26 \\
7. La ciencia contribuye a mejorar la calidad de vida & $\mathbf{0 . 6 6}$ & 0.44 \\
8. Nuestro país está gastando demasiado dinero en la ciencia (i) & $\mathbf{0 . 5 7}$ & 0.33 \\
9. La ciencia puede ayudar a hacer del mundo un lugar mejor & $\mathbf{0 . 5 0}$ & 0.25 \\
10. El dinero utilizado en proyectos científicos es dinero desperdiciado (i) & 2.86 \\
Valores propios (eigenvalues) & - \\
\hline b2 & & -
\end{tabular}

$h^{2}$ refiere a las comunalidades de los ítems

(i) refiere a los ítems cuyos resultados deben ser invertidos antes del análisis

- refiere a los ítems que han sido eliminados para el análisis de componentes principales de la versión definitiva del instrumento 